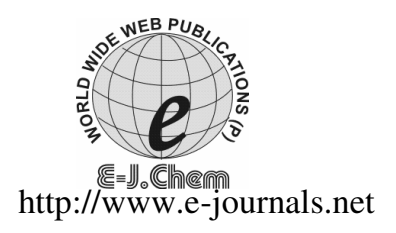

ISSN: 0973-4945; CODEN ECJHAO

E-Journal of Chemistry

$2011,8(3), 1146-1151$

\title{
Ultrasonic Studies of Some Biomolecules in Aqueous Guanidine Hydrochloride Solutions at 298.15 K
}

\author{
R. PALANI ${ }^{*}$, A. GEETHA ${ }^{\#}$ and S.V. S. L. POORNIMA \\ Department of Physics, D.D.E, Annamalai University \\ Annamalainagar 608 002, Tamil Nadu, India \\ \#Department of Physics, SRM University \\ Kattankulathur, Kancheepuram, 603203, India \\ palani_physics06@yahoo.co.in
}

Received 27 October 2010; Accepted 20 January 2011

\begin{abstract}
Ultrasonic velocity $(U)$, viscocity $(\eta)$ and density $(\rho)$ of $L$ - ascorbic acid, dextrose and sucrose in aqueous guanidine hydrochloride $(0.3 \mathrm{~m})$ have been measured at $298.15 \mathrm{~K}$. The derived acoustical parameters namely adiabatic compressibility $(\beta)$, apparent molal compressibility $\left(\varphi_{\mathrm{K}}\right)$ apparent molal volume $\left(\varphi_{\mathrm{v}}\right)$ limiting apparent molal compressibility $\left(\left(\varphi_{\mathrm{K}}^{0}\right)\right.$ limiting apparent molal volume $\left(\varphi_{\mathrm{v}}^{0}\right)$ and their constants $\left(\mathrm{S}_{\mathrm{K}}, \mathrm{S}_{\mathrm{V}}\right)$ and viscosity $B$ coefficient and hydration number $\left(n_{H}\right)$ have been computed from the experimental data. The variation of these parameters with respect to the molality of solutes have been explained on the basis of solute-solvent interaction and structure making tendency of the solutes in solvents.
\end{abstract}

Keywords: Ultrasonic velocity, Hydration number, Adiabatic compressibility, Apparent molal compressibility, Apparent molal volume.

\section{Introduction}

Ultrasonic investigation provides an extensive application in characterizing thermodynamic and physicochemical behavior of liquid mixtures. Ultrasonic measurements are very useful in industry (chemical and food processing), medicine, material testing (flaw detection) and cleaning (Ultrasonic bath). The measurement of some useful acoustical parameters which are highly sensitive to the study of molecular interactions. These acoustical parameters provide qualitative information about the physical nature and strength of the molecular interactions in the liquid mixtures. During the last two decades, the ultrasonic study of the carbohydrates in aqueous electrolytic medium, has gained much importance in assessing the nature of molecular interaction present in the mixture. The study of the carbohydrates / saccharides has become a subject of increasing interest because of the multidimensional, physical, biochemical and industrially useful properties of these compounds ${ }^{1-4}$, in addition to their importance to the food pharmaceutical and chemical industries, the simple saccharides have received considerable attention for their ability to protect biological macromolecules ${ }^{5}$. 
In recent years, the study of acoustical properties of aqueous mixed electrolytic solutions has been found to be useful in understanding the specific ion-ion and ion-solvent interaction in solutions. Such studies on binary electrolytes have been made by many researchers ${ }^{6-7}$. However ultrasonic velocity studies on ternary electrolytes have not received as much attention as that of binary electrolytes. Since, physicochemical studies of binary electrolytes cannot be so useful to get a definite conclusion regarding the structural properties and the type of interaction involved the study of ternary electrolytes in gaining much importance now- $a$-days. Ionic association and electrostatic interactions are the prime factors that must be considered in these electrolytes. Further, the flexibility of saccharide molecules is considerable relevance for their interactions with metal cations. Guanidine hydrochloride is stronger denaturating agent than urea, therefore the study of interactions between guanidine hydrochloride $(\mathrm{GuHCl})$ and saccharides will throw further light on the hydration characteristics of the saccharides which will help in understanding the mechanism of protein stabilization. Owing to these considerations an attempt has been made to elucidate the molecular associations / interactions of three component liquid mixtures of $L$-ascorbic acid, dextrose and sucrose in aqueous guanidine hydrochloride $(0.3 \mathrm{~m})$ at $298.15 \mathrm{~K}$ by measuring ultrasonic velocity, density and viscosity. From the experimental values, the acoustical parameters such as adiabatic compressibility, apparent molal compressbility, apparent molal volume, limiting apparent molal compressibility, limiting apparent molal volume and their constants $S_{K}$ and $S_{V}$ viscosity B coefficients of Jone - Doles equation and molal hydration number have been computed to shed more light on such interactions.

\section{Experimental}

All the chemicals used in this present research work are spectroscopic reagent (SR) and analytical reagent (AR) grades of minimum assay of $99.9 \%$ obtained from E-Merck, Germany and Sd fine chemicals, India, which are used as such without further purification. Doubly distilled deionised water has been used for preparing aqueous $\mathrm{GuHCl}$ mixtures. Required amount of water and $\mathrm{GuHCl}$ were taken to prepare the composition of $0.3 \mathrm{~m}$ binary mixtures in a clean dry conical flask with ground stopper. The required quantity of biomolecules for given molality was dissolved and similar procedure has been adopted for different molalities of the same. All the solutions were prepared by weight using an electronic digital balance having an accuracy of $\pm 0.1 \mathrm{mg}$. (Model: SHIMADZU AX-200). The density was determined using a specific gravity bottle by relative measurement method with an accuracy of $\pm 0.1 \mathrm{~kg} \cdot \mathrm{m}^{-3}$. An Ostwald's viscometer (10 mL capacity) was used for the viscosity measurement and efflux time was determined using a digital chronometer within $\pm 0.01 \mathrm{~s}$. An ultrasonic interferometer having the frequency $3 \mathrm{MHz}$ (Mittal Enterprises, New Delhi, Model: F81) with an overall accuracy of $\pm 0.1 \%$ has been used for velocity measurement. An electronically digital operated constant temperature bath (RAAGA Industries) has been used to circulate water through the double walled measuring cell made up of steel containing the experimental solution at the desired temperature. The accuracy in the temperature measurement is $\pm 0.1 \mathrm{~K}$.

\section{Theory and calculations}

Various acoustical and thermodynamical parameters are calculated from the measured data such as

Adiabatic compressibility

$$
\beta=\frac{1}{U^{2} \rho}
$$


The apparent molal compressibility has been calculated from relation,

$$
\varphi_{K}=\frac{1000}{m \rho_{0}}\left(\rho_{0} \beta-\rho \beta_{0}\right)+\left(\frac{\beta_{0} M}{\rho_{0}}\right)
$$

Where $\beta, \rho$ and $\beta_{0}, \rho_{0}$ are the adiabatic compressibility and density of solution and solvent respectively, $\mathrm{m}$ is the molal concentration of the solute and $\mathrm{M}$ the molecular mass of the solute. $\varphi_{\mathrm{K}}$ is the function of $\mathrm{m}$ as obtained by Gucker ${ }^{8}$ from Debye Huckel ${ }^{9}$ theory and is given by

$$
\varphi_{K}=\varphi_{K}{ }^{0}+S_{K} m^{1 / 2}
$$

Where $\varphi_{\mathrm{K}}{ }^{0}$ is the limiting apparent molal compressibility at infinite dilution and $\mathrm{S}_{\mathrm{K}}$ is a constant. $\varphi_{\mathrm{K}}{ }^{0}$ and $\mathrm{S}_{\mathrm{K}}$ of equation (3) have been evaluated by the least square method. The apparent molal volume $\varphi_{\mathrm{V}}$ has been calculated using the relation

$$
\varphi_{V}=\left(\frac{M}{\rho}\right)-\frac{1000\left(\rho-\rho_{0}\right)}{m \rho \rho_{0}}
$$

The apparent molal volume $\varphi_{\mathrm{V}}$ has been found to differ with concentration according to Masson ${ }^{10}$ empirical relation as

$$
\varphi_{V}=\varphi_{V}^{0}+S_{V} m^{1 / 2}
$$

Where $\varphi_{V}^{0}$ is the limiting apparent molal volume at infinite dilution and $S_{V}$ is a constant and these values were determined by least square method. The viscosity A and B coefficients for the lactose in aqueous electrolytic solutions are calculated from the Jones-Dole ${ }^{11}$ equation.

$$
\frac{\eta}{\eta_{0}}=1+A m^{1 / 2}+B m
$$

Where, $\eta$ and $\eta_{0}$ are the viscosities of the solution and solvent respectively and $m$ is the molal concentration of the solute. A is determined by the ionic attraction theory of falkenhagen-vernon ${ }^{12}$ and therefore also called falkenhagen coefficient, B or Jones-Dole coefficient is an empirical constant determined by solute-solvent interactions. The molal hydration number has been computed using the relation,

$$
\mathrm{n}_{\mathrm{H}}=\left(\frac{\mathrm{n}_{1}}{\mathrm{n}_{2}}\right)\left(1-\frac{\beta}{\beta_{0}}\right)
$$

Where $\beta$ and $\beta_{0}$ are adiabatic compressibility of solution and solvent respectively, $\mathrm{n}_{1}$ and $\mathrm{n}_{2}$ are number of moles of solvent and solute respectively.

\section{Results and Discussion}

The experimental values of density, viscosity and ultrasonic velocity for different molal composition of $L$-ascorbic acid, dextrose and sucrose in aqueous $\mathrm{GuHCl}$ mixtures $(0.3 \mathrm{~m})$ are presented in Table 1. The values of adiabatic compressibility, apparent molal compressibility, apparent molal volume and hydration number $\left(n_{H}\right)$, limiting apparent molal compressibility, limiting apparent molal volume and their constant $S_{K}$ and $S_{V}$ the values of $A$ and $B$-coefficients of Jone-Doles equations and are presented in Tables 2 and 3. 
In all the three system (Table 1) the values of density and ultrasonic velocity are increases with increase in molal concentration of saccharides and $L$-ascorbic acid at a given temperature. This increasing trend suggests a moderate strong electrolytic nature in which the solutes tend to attract the solvent molecules. Molecular interactions are thus responsible for the observed increase in ultrasonic velocity in these mixtures. The increase in ultrasonic velocity in these solutions may be attributed to the cohesion brought about by the ionic hydration.

Table 1. Values of density ( $\rho)$, viscosity $(\eta)$ and ultrasonic velocity (U) of $L$-ascorbic acid, dextrose and sucrose in aqueous guanidine hydrochloride solution at $298.15 \mathrm{~K}$

\begin{tabular}{|c|c|c|c|}
\hline Molality $\mathrm{m} / \mathrm{mol} . \mathrm{kg}^{-1}$ & $\rho / \mathrm{kg} \cdot \mathrm{m}^{-3}$ & $\eta / \times 10^{-3} \mathrm{Nsm}^{-2}$ & $\mathrm{U} / \mathrm{ms}^{-1}$ \\
\hline \multicolumn{4}{|c|}{ System I : Water+guanidine hydrochloride $+L$-ascorbic acid } \\
\hline 0.0 & 1009.0 & 0.9333 & 1560.0 \\
\hline 0.2 & 1022.0 & 0.9874 & 1544.5 \\
\hline 0.4 & 1031.2 & 1.0212 & 1555.6 \\
\hline 0.6 & 1055.3 & 1.1012 & 1560.8 \\
\hline 0.8 & 1056.0 & 1.1491 & 1567.3 \\
\hline 1.0 & 1068.4 & 1.2328 & 1571.7 \\
\hline \multicolumn{4}{|c|}{ System II : Water+ guanidine hydrochloride +dextrose } \\
\hline 0.0 & 1009.0 & 0.9333 & 1560.0 \\
\hline 0.2 & 1026.9 & 0.9889 & 1545.6 \\
\hline 0.4 & 1034.9 & 1.0578 & 1557.7 \\
\hline 0.6 & 1058.2 & 1.1149 & 1573.6 \\
\hline 0.8 & 1061.5 & 1.1754 & 1575.9 \\
\hline 1.0 & 1070.03 & 1.5504 & 1582.5 \\
\hline \multicolumn{4}{|c|}{ System III : Water+ guanidine hydrochloride +sucrose } \\
\hline 0.0 & 1009.0 & 0.9333 & 1560.0 \\
\hline 0.2 & 1036.7 & 1.0520 & 1548.4 \\
\hline 0.4 & 1056.4 & 1.1837 & 1563.7 \\
\hline 0.6 & 1081.2 & 1.6473 & 1585.0 \\
\hline 0.8 & 1093.2 & 1.8547 & 1592.7 \\
\hline 1.0 & 1112.6 & 2.4744 & 1619.7 \\
\hline
\end{tabular}

The decrease in adiabatic compressibility (Table 2), observed in the present study generally confirms that conclusion drawn from the velocity data. The increasing electrostrictive compression of water around the molecules results in a large decrease in the compressibility of solutions. The decrease in compressibility implies that there is enhanced molecular association in these systems an increase solute content, as the new entities (formed due to molecular association) become compact and less compressible ${ }^{13}$. The increase in velocity and decrease in compressibility were attributed to the formation of hydrogen bonds between solute and solvent molecules.

From the Table 2, it is observed that the positive hydration number increases non linearly with solutes which further confirms the existence of molecular interaction between solute and solvent molecules.

Further, from the Table it is observed that

(i) The values of $\varphi_{\mathrm{K}}$ and $\varphi_{\mathrm{V}}$ are all negative over the entire range of molality of solutes and it varies non linearly.

(ii) The maximum value of $\varphi_{\mathrm{V}}$ obtained in all the systems is in the order: 
Table 2. Values of adiabatic compressibility $(\beta)$, hydration number $\left(n_{H}\right)$, apparent molal compressibility $\left(\varphi_{\mathrm{K}}\right)$ and apparent molal volume $\left(\varphi_{\mathrm{V}}\right)$ of $L$-ascorbic acid, dextrose and sucrose in aqueous guanidine hydrochloride solution at $298.15 \mathrm{~K}$

\begin{tabular}{|c|c|c|c|c|}
\hline $\begin{array}{c}\text { Molality } \\
\mathrm{m} / \mathrm{mol} \mathrm{kg}^{-1}\end{array}$ & $\beta / \times 10^{-10} \mathrm{~m}^{2} \mathrm{~N}^{-1}$ & $\left(\mathrm{n}_{\mathrm{H}}\right)$ & $-\varphi_{\mathrm{K}} / \times 10^{-8} \mathrm{~m}^{2} \mathrm{~N}^{-1}$ & $-\varphi_{\mathbf{V}} / \mathrm{m}^{3} \mathrm{~mol}^{-1}$ \\
\hline \multicolumn{5}{|c|}{ System I : Water+guanidine hydrochloride $+L$-ascorbic acid } \\
\hline 0.0 & 4.0720 & $\ldots$ & $\ldots$ & $\ldots$ \\
\hline 0.2 & 4.1017 & -1.9 & 1.14 & 64.42 \\
\hline 0.4 & 4.0070 & 2.2 & 3.86 & 55.00 \\
\hline 0.6 & 3.8800 & 4.2 & 6.31 & 76.48 \\
\hline 0.8 & 3.8550 & 3.1 & 5.08 & 58.22 \\
\hline 1.0 & 3.7890 & 3.8 & 5.22 & 58.86 \\
\hline \multicolumn{5}{|c|}{ System II : Water+ guanidine hydrochloride +dextrose } \\
\hline 0.0 & 4.072 & $\ldots$ & $\ldots$ & $\ldots$ \\
\hline 0.2 & 4.076 & -0.2 & 3.41 & 88.70 \\
\hline 0.4 & 3.981 & 3.0 & 4.89 & 64.15 \\
\hline 0.6 & 3.816 & 5.6 & 7.58 & 81.26 \\
\hline 0.8 & 3.793 & 4.5 & 6.14 & 65.04 \\
\hline 1.0 & 3.731 & 4.4 & 5.87 & 60.48 \\
\hline \multicolumn{5}{|c|}{ System III : Water+ guanidine hydrochloride +sucrose } \\
\hline 0.0 & 4.072 & $\ldots$ & $\ldots$ & $\ldots$ \\
\hline 0.2 & 4.023 & 0.3 & 8.03 & 137.26 \\
\hline 0.4 & 3.871 & 6.6 & 9.81 & 117.44 \\
\hline 0.6 & 3.681 & 8.5 & 11.38 & 119.37 \\
\hline 0.8 & 3.606 & 7.6 & 10.07 & 104.30 \\
\hline 1.0 & 3.426 & 8.4 & 10.64 & 102.67 \\
\hline
\end{tabular}

A non-linear variation of negative $\varphi_{\mathrm{K}}$ and $\varphi_{\mathrm{V}}$ values with respect to the solute concentration in all the systems studied which indicates an existence of solute - solvent interaction. From the magnitude of $\varphi_{\mathrm{V}}$, it can be concluded that, strong molecular interaction is found in sucrose mixture and hence sucrose mixture is a more effective structure maker than others.

From the Table 3 it is observed that $\varphi_{\mathrm{K}}^{0}$ values are positive in all systems studied. Appreciable positive values of $\varphi_{\mathrm{K}}^{0}$ for all systems reinforce that the ionic-hydrophilic interactions are dominating over the ionic- hydrophobic interactions. Therefore the mutual overlap of the hydration co spheres of solute and co solute molecules will lead to an increase in the magnitude of hydrogen bonding interactions between - $\mathrm{NH}$ groups of $\mathrm{GuHCl}$ and $-\mathrm{OH}$ groups of saccharide molecules. The values of $S_{K}$ exhibits negative in all systems studied which indicates the existence of solute-solute interactions. From the Table 3 it is observed that the values of $\varphi_{\mathrm{v}}^{0}$ in all the systems are negative. This enhances / reduces the electrostriction of water molecules. The negative values of $\varphi_{\mathrm{v}}^{0}$ indicate smaller solute-solvent interactions present in these systems. The magnitude of $\varphi_{\mathrm{v}}^{0}$ is the order: sucrose $<$ dextrose $<L$-ascorbic acid. It is evident from the Table 3 that $S_{\mathrm{V}}$ exhibits positive values in all the systems, suggesting the presence of strong ion-ion / solute - solute interaction in the mixtures.

From the Table 1 it is observed that the values of viscosity increases with increase in concentration of solutes. This increasing trend indicates the existence of molecular interaction occurring in these systems. In order to shed more light on this, the role of viscosity $B$-coefficient has been obtained. From the Table 3, it is observed that the values of A are negative in all systems studied and $B$-coefficient are positive. 
Table 3. Values of limiting apparent molal compressibility $\left(\varphi_{\mathrm{K}}^{0}\right)$, limiting apparent molal volume $\left(\varphi_{\mathrm{v}}^{0}\right)$, constants $S_{\mathrm{K}}$ and $\mathrm{S}_{\mathrm{V}}$ and $\mathrm{A}$ and $B$ - coefficients of Jones-Dole equation of $L$-ascorbic acid, dextrose and sucrose in aqueous guanidine hydrochloride solution at $298.15 \mathrm{~K}$

\begin{tabular}{lcccccc}
\hline $\begin{array}{c}\text { Aqueous guanidine } \\
\text { Hydrochloride } \\
\text { solutions }\end{array}$ & $\begin{array}{c}\varphi_{\mathrm{k}}^{0} / \times 10^{-8} \\
\mathrm{~m}^{2} \mathrm{~N}^{-1}\end{array}$ & $\begin{array}{c}-\mathrm{S}_{\mathrm{k}} /\left(\times 10^{-8}\right. \\
\mathrm{Nol}^{-1} \mathrm{~m}^{-1}\end{array}$ & $\begin{array}{c}-\varphi_{\mathrm{v}}^{0} / \mathrm{m}^{3} . \\
\mathrm{mol}^{-1}\end{array}$ & $\begin{array}{c}\mathrm{S}_{\mathrm{v}} / \mathrm{m}^{3} \mathrm{lt}^{1 / 2} \\
\mathrm{~mol}^{-3 / 2}\end{array}$ & $\begin{array}{c}\mathrm{A} / \mathrm{dm}^{3 / 2} \\
\mathrm{~mol}^{-1 / 2}\end{array}$ & $\begin{array}{c}\mathrm{B} / \mathrm{dm}^{3} \\
\mathrm{~mol}^{-1}\end{array}$ \\
\hline $\begin{array}{l}L \text {-ascorbic acid } \\
\text { Dextrose }\end{array}$ & 5.56 & 66.07 & 7.416 & 14.63 & -0.046 & 0.352 \\
Sucrose & 3.68 & 102.74 & 4.909 & 41.10 & -0.081 & 0.458 \\
\hline
\end{tabular}

Since $\mathrm{A}$ is a measure of ionic interaction ${ }^{14}$ it is evident that there is a strong solute - solute interaction in the mixtures studied, which is indicated by the smaller magnitude of A values. $B$ - coefficient is also known as measure of order and disorder introduced by the solute into the solvent. It is also a measure of solute - solvent interaction and relative size of the solute and solvent molecules. The behavior of $B$ - coefficient in all the three systems suggests the existence of strong ion - solvent interaction. The magnitude of $\mathrm{B}$ values is in the order of sucrose $>$ dextrose $>$ $L$ - ascorbic acid. This conclusion is an excellent agreement with that drawn from $S_{\mathrm{V}}$ and $\varphi_{\mathrm{V}}^{0}$ data and the larger values of B indicate structure making capacity of the solute.

\section{Conclusion}

The present study reports experimental data for density, viscosity and ultrasonic velocity at $298.15 \mathrm{~K}$ for $L$-ascorbic acid, dextrose and sucrose in aqueous guanidine hydrochloride mixture. From these data, several acoustical parameters have been calculated and studied to explain the intermolecular interactions between mixing components. The ionic - hydrophilic type of interactions are operating between $\mathrm{GuHCl}$ and studied biomolecules. The existence of solute - solvent and solute-solute interactions resulting in attractive forces promote the structure making tendency of the solute and solvent molecules. The existence of molecular interaction is in the order of sucrose $>$ dextrose $>L$-ascorbic acid. This suggests sucrose in aqueous $\mathrm{GuHCl}$ mixture is a strong structure maker than other two mixtures.

\section{References}

1. Golberg R N and Tewari Y B, J Bio Chem., 1989, 264(17), 9897-9900.

2. Boerio Goates J, J Chem Thermodyn., 1991, 23, 403.

3. Tewari Y B and Golberg R N, Biophy Chem., 1991, 40, 59.

4. Birch G G and Shamil S, J Chem Soc Faraday Tran., 1998, 1, 84(8), 2635-2640.

5. Banipal T S, Damanjit Kaur, Gagandeep Singh, Lark B S and Banipal P K, Indian J Chem., 2002, 41A, 1131-1138.

6. $\quad$ Pakade S V and Yawale S P, J Pure Appl Ultrason., 1996, 18, 74-79.

7. Shree Krishna Kumar K, Jugan J and Roshan Abraham, J Pure Appl Ultrason., 1997, 19, 32.

8. Debye P and Huckel E, Physik Z, 1923, 24, 185.

9. Gucker B S, Chauhan S and Syal V K, Indian J Chem., 1933, 41A, 111.

10. Masson D O, Philos Mag., 1929, 8, 218.

11. Jones G and Dole M, J Am Chem Soc., 1929, 51, 2950.

12. Falkenhagen H and Vernan E L, Z Phys., 1932, 33, 140.

13. Fort R J and Moore W R, Trans Faraday Soc., 1965, 61, 2102.

14. Jahagiridhar D V, Arbad B R, Patil S C S and Shankarwar A G, Indian J Pure Appl Phy., 2000, 38, 645. 


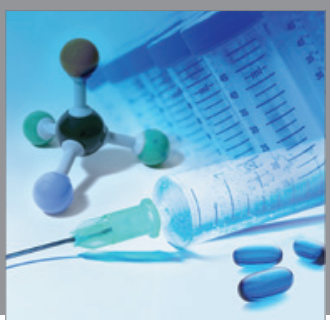

International Journal of

Medicinal Chemistry

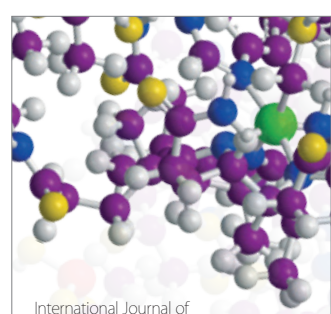

Carbohydrate Chemistry

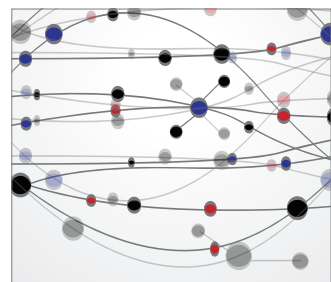

The Scientific World Journal
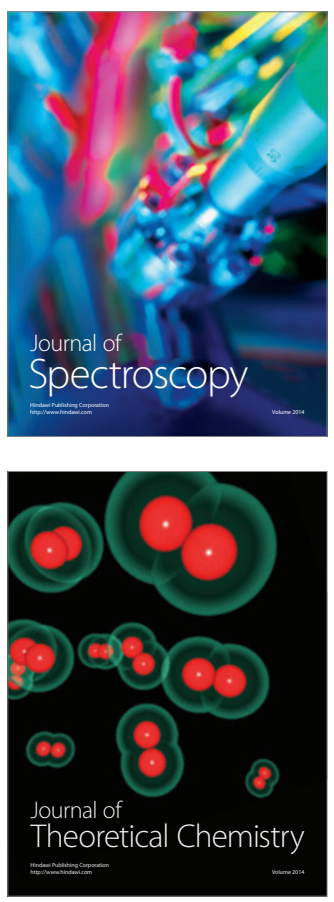
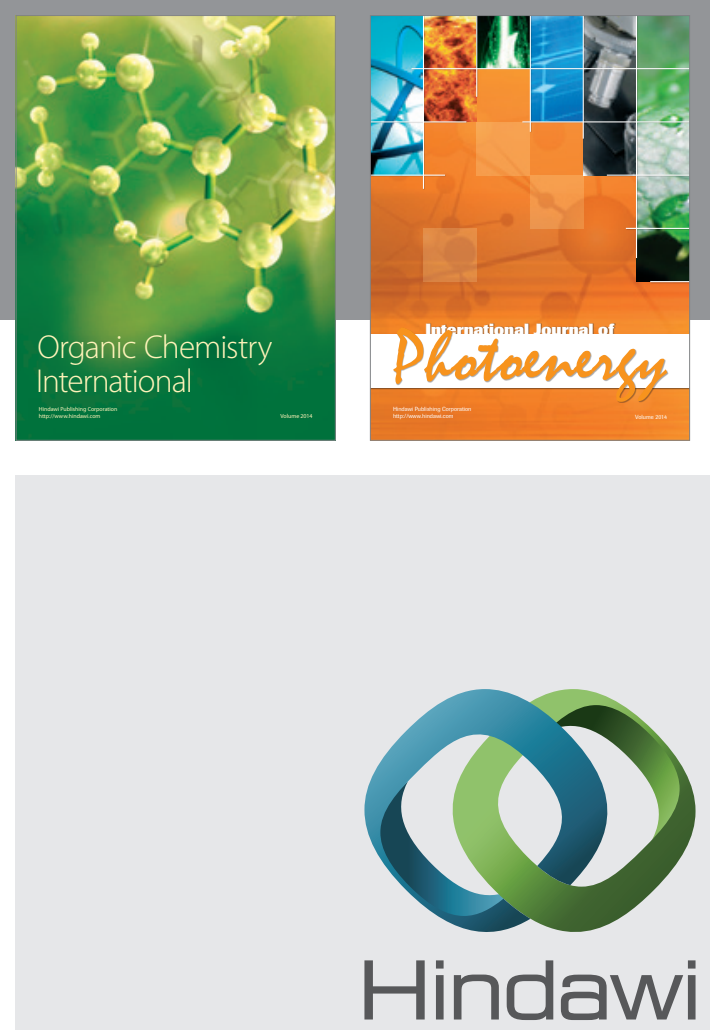

Submit your manuscripts at

http://www.hindawi.com
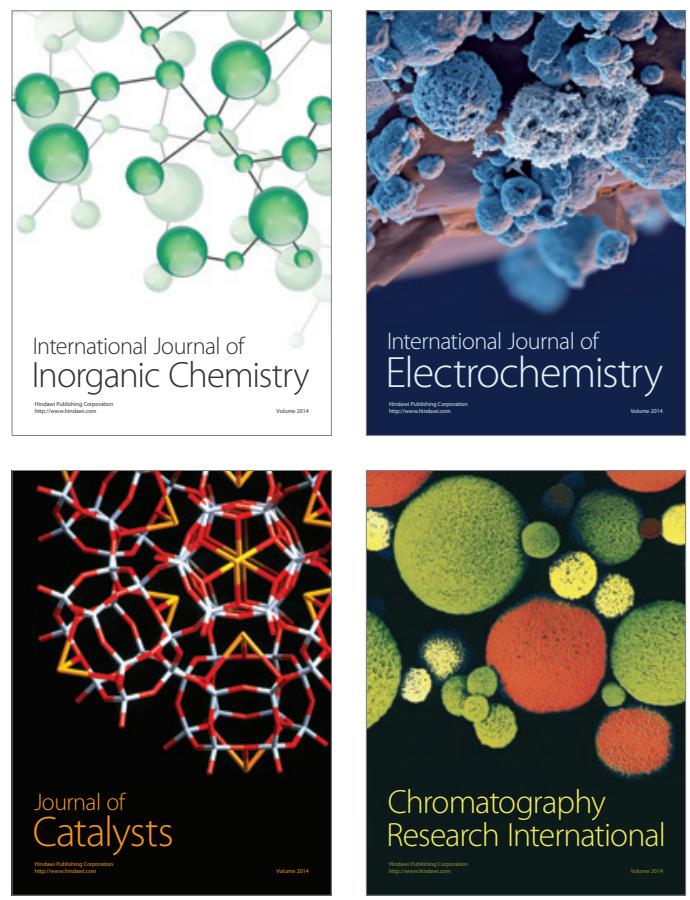
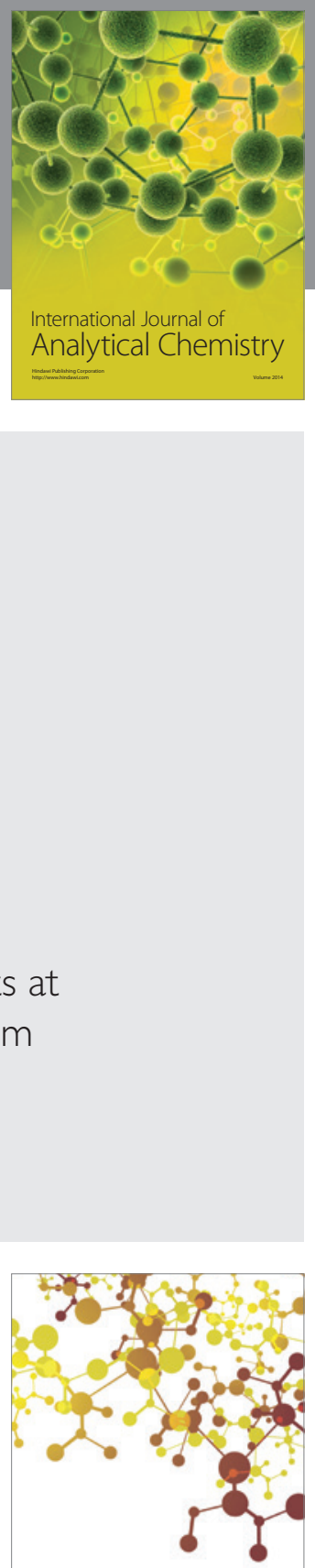

Journal of

Applied Chemistry
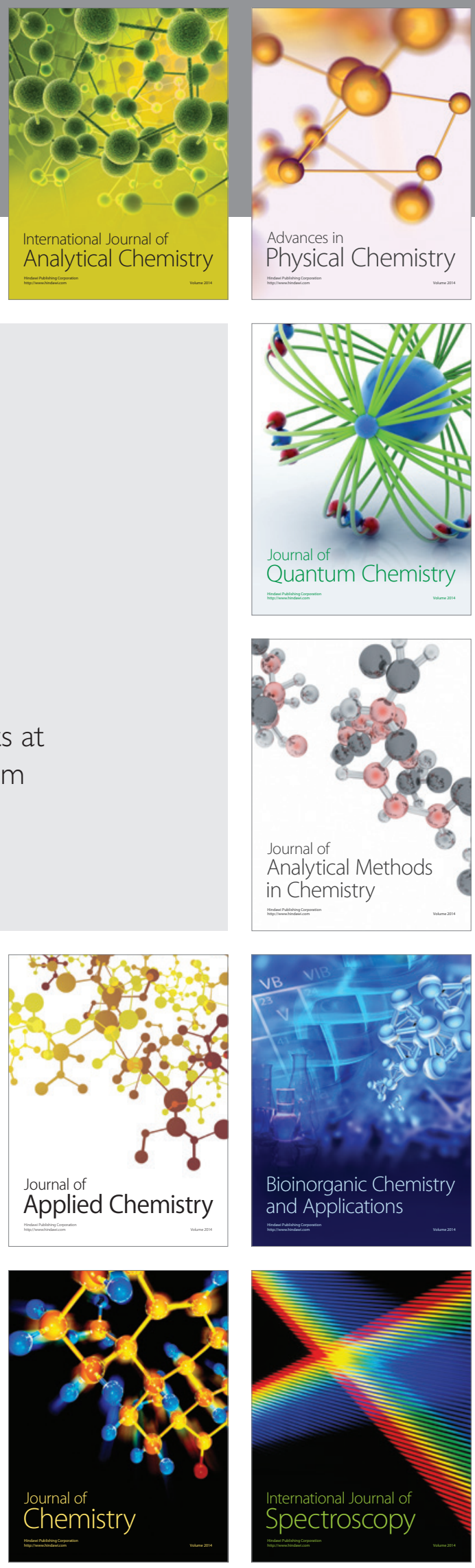